\title{
Segments in enumerating faces
}

\author{
Katta G. Murty ${ }^{\mathrm{a}, *}$, Sung-Jin Chung ${ }^{\mathrm{b}, 1}$ \\ ${ }^{a}$ Department of Industrial and Operations Engineering, University of Michigan, \\ Ann Arbor, MI 48109-2117, USA \\ ${ }^{\mathrm{b}}$ Department of Industrial Engineering, Seoul National University, Seoul, South Korea
}

Received 26 April 1993; revised manuscript received 10 January 1994

\begin{abstract}
We introduce the concept of a segment of a degenerate convex polytope specified by a system of linear constraints, and explain its importance in developing algorithms for enumerating the faces. Using segments, we describe an algorithm that enumerates all the faces, in time polynomial in their number. The role of segments in the unsolved problem of enumerating the extreme points of a convex polytope specified by a degenerate system of linear constraints, in time polynomial in the number of extreme points, is discussed.
\end{abstract}

Keywords: Convex polytopes; Enumeration of faces; Adjacency; Segments

\section{Introduction}

The problem of enumerating all the extreme points (or vertices) of a convex polytope specified by a system of linear constraints has been studied extensively in the literature. It is discussed in textbooks (see [14, Section 3.19]), and a large number of journal articles have discussed a variety of algorithms for it (see $[1-6,9,10,12,16,17]$ ). There are some applications in which this problem appears, but the typical exponential growth of the number of extreme points in terms of the number of variables $n$ and the number of constraints $\rho$ in the linear system describing the polytope makes this practical only for systems in which both $n$ and $\rho$ are small. In spite of this, there is considerable mathematical interest in developing efficient new algorithms for this problem and the problem of enumerating not only the extreme points, but all the faces of all dimensions.

\footnotetext{
${ }^{*}$ Corresponding author. e-mail: murty@engin.umich.edu.

${ }^{1}$ Work carried out while on sabbatical leave in the Industrial and Operations Engineering Department at the University of Michigan in Ann Arbor, USA.
} 
For any matrix $H$, we denote by $H_{i}, H_{. j}$, its $i$ th row vector and $j$ th column vector, respectively. We will use the abbreviation LP for "linear program". For two sets $D, E$, $\boldsymbol{D} \backslash \boldsymbol{E}$ denotes the set of elements of $\boldsymbol{D}$ which are not in $\boldsymbol{E}$.

If $\left\{a^{1}, \ldots, a^{t}\right\}$ is a set of points in $\mathbb{R}^{n}$, we denote its convex hull by $\left\langle a^{1}, \ldots, a^{t}\right\rangle$. The affine rank of $\left\{a^{1}, \ldots, a^{t}\right\}$ is defined to be the rank of the set $\left\{a^{2}-a^{1}, \ldots, a^{t}-a^{1}\right\}$; it is the dimension of the affine space of $\left\{a^{1}, \ldots, a^{t}\right\}$.

Without any loss of generality, we consider the convex polytope $\boldsymbol{K}$ which is the set of feasible solutions of the system of constraints

$$
A x=b, \quad x \geqslant 0,
$$

where $A$ is a matrix of order $m \times n$ and $\operatorname{rank} m$, and $n>m$. For $j=1, \ldots, n, A_{. j}$ is the column of the variable $x_{j}$ in (1). Our method can be extended very directly to handle polytopes defined by more general systems consisting of linear equations, inequalities and/or bounds on variables; or such general systems can be transformed into a system of the form (1) by simple transformations that preserve one-to-one correspondence between faces of the original and the transformed systems. We assume that $A, b$ are integer, and that $\boldsymbol{K}$ is nonempty and bounded.

If $\max \left\{x_{j}: x \in \boldsymbol{K}\right\}=0$, then the variable $x_{j}$ is equal to the constant 0 all over $\boldsymbol{K}$ and can be eliminated. So, we assume that $\max \left\{x_{j}: x \in \boldsymbol{K}\right\}>0$ for all $j=1, \ldots, n$. This implies that the dimension of $\boldsymbol{K}$ is $n-m$.

Given a convex polytope $\boldsymbol{\Gamma} \subset \mathbb{R}^{n}$, a hyperplane $\boldsymbol{H}$ in $\mathbb{R}^{n}$ is said to be a supporting hyperplane for $\boldsymbol{\Gamma}$ iff $\boldsymbol{\Gamma}$ is completely contained in one of the halfspaces determined by $\boldsymbol{H}$, and $\boldsymbol{\Gamma} \cap \boldsymbol{H} \neq \emptyset$.

Definition 1.1 (Faces of a convex polytope). Let $\Gamma \subset \mathbb{R}^{n}$ be a convex polytope. A face of $\boldsymbol{\Gamma}$ is either the empty set, or $\boldsymbol{\Gamma}$ itself, or the intersection of $\boldsymbol{\Gamma}$ with a supporting hyperplane.

Faces of dimension 0 of a convex polytope are its extreme points or vertices, and its one-dimensional faces are its edges. A facet of a convex polytope $\Gamma$ is a face whose dimension is one less than the dimension of $\boldsymbol{\Gamma}$. A facetal hyperplane of a fulldimensional convex polytope in $\mathbb{R}^{n}$ is a hyperplane in $\mathbb{R}^{n}$ containing one of its facets.

Geometrically, an extreme point of a convex polytope is said to be nondegenerate (degenerate) if the number of facets incident at it is equal to (strictly greater than) the dimension of the convex polytope. The convex polytope itself is said to be nondegenerate if all its extreme points are nondegenerate, degenerate if at least one of its extreme points is degenerate.

Given a linear inequality constraint and a point $\bar{x}$ satisfying it, the constraint is said to be active at $\bar{x}$ if it holds as an equation there, inactive if it holds as a strict inequality.

Given a convex polytope $\boldsymbol{\Gamma}$ in $\mathbb{R}^{n}$, it can be represented algebraically through a system of linear equations and inequalities in variables $x=\left(x_{1}, \ldots, x_{n}\right)^{\mathrm{T}}$, in several different ways. The various representations differ in redundant constraints, etc. Let (P) denote a linear constraint representation of $\boldsymbol{\Gamma}$, in which if there are any linear 
equations, the system of linear equations is linearly independent. In this representation (P), algebraically, an extreme point $\bar{x}$ of $\boldsymbol{\Gamma}$ is said to be nondegenerate (degenerate) if the total number of linear equations in $(\mathrm{P})$ and the inequality constraints in $(\mathrm{P})$ active at $\bar{x}$ is equal to $n$ (strictly greater than $n$ ). The system (P) is said to be nondegenerate if every extreme point of $\boldsymbol{\Gamma}$ is nondegenerate; degenerate if at least one extreme point of $\boldsymbol{\Gamma}$ is degenerate by this algebraic definition.

If a convex polytope is degenerate (nondegenerate) by the geometrical definition, every minimal representation (i.e., a representation using the smallest possible number of constraints) of it will be degenerate (nondegenerate). Also, when a system representing a convex polytope is nondegenerate, the convex polytope is nondegenerate.

From the definition we see that system (1) representing $K$ is degenerate if $b$ is in the nonnegative hull of some set of $m-1$ or less column vectors of $A$, nondegenerate otherwise. The problem discussed in this paper is to enumerate all the faces of $\boldsymbol{K}$ when it is represented by a system of the form (1) which is degenerate.

Some methods for enumerating faces use a pivot scheme to first enumerate the extreme points of $\boldsymbol{K}$ based on enumerating the feasible bases for (1). Every extreme point of $\boldsymbol{K}$ is the basic feasible solution (BFS) associated with one or more feasible bases for (1); this is the principle used in these methods.

If (1) is nondegenerate, every extreme point of $\boldsymbol{K}$ is associated with a unique feasible basis for (1) and vice versa. If $\ell_{0}$ is the number of extreme points of $K$, the pivot scheme of [2] enumerates all these extreme points in time $\mathrm{O}\left(\ell_{0} m n\right)$, an effort which grows linearly with $\ell_{0}$. Also, in this case the pivot step of entering any nonbasic variable into a feasible basic vector leads to an edge. Hence in this case at every vertex of $\boldsymbol{K}$, the number of incident edges is equal to the dimension of $\boldsymbol{K}$, that is why $\boldsymbol{K}$ is said to be a simple (or regular) convex polytope in this case, and all its edges can be generated with an effort which grows linearly with their number by the pivot scheme of entering one nonbasic variable at a time into a feasible basic vector for (1). And when (1) is nondegenerate, for any $r$, any set of $r$ edges $\left\{e_{1}, \ldots, e_{r}\right\}$ of $\boldsymbol{K}$ with a common vertex $\bar{x}$ defines an $r$-dimensional face $\boldsymbol{F}$ containing that vertex $\bar{x}$, with the property that $\left\{e_{1}, \ldots, e_{r}\right\}$ is exactly the set of edges incident at $\bar{x}$ in $\boldsymbol{F}$ [13]. So, in this case, once all the edges are determined, all faces of all dimensions can be determined using this result, in linear time [8].

If (1) is degenerate, there may be several feasible bases associated with a degenerate extreme point of $\boldsymbol{K}$, and the number of feasible bases for (1) may grow exponentially with $\ell_{0}$ and $n$. A degenerate polytope will have at least one vertex at which the number of incident edges is strictly greater than the dimension of the polytope, hence such polytopes are also called nonsimple polytopes. And in this case, for $r \geqslant 2$, given a set $\boldsymbol{E}$ of $\boldsymbol{r}$ edges incident at a vertex $\bar{x}$ of $\boldsymbol{K}$, there may be no face of $\boldsymbol{K}$ containing exactly these edges incident at $\bar{x}$. As an example, consider the polytope in $\mathbb{R}^{3}$ illustrated in Fig. 1. On this polytope, the pair of edges (one joining $p^{4}$ and $p^{3}$ and the other joining $p^{4}$ and $p^{2}$ ) do not form a two-dimensional face, even though they have a common vertex $p^{4}$.

In [16], Provan developed a polynomial time algorithm for enumerating the extreme 


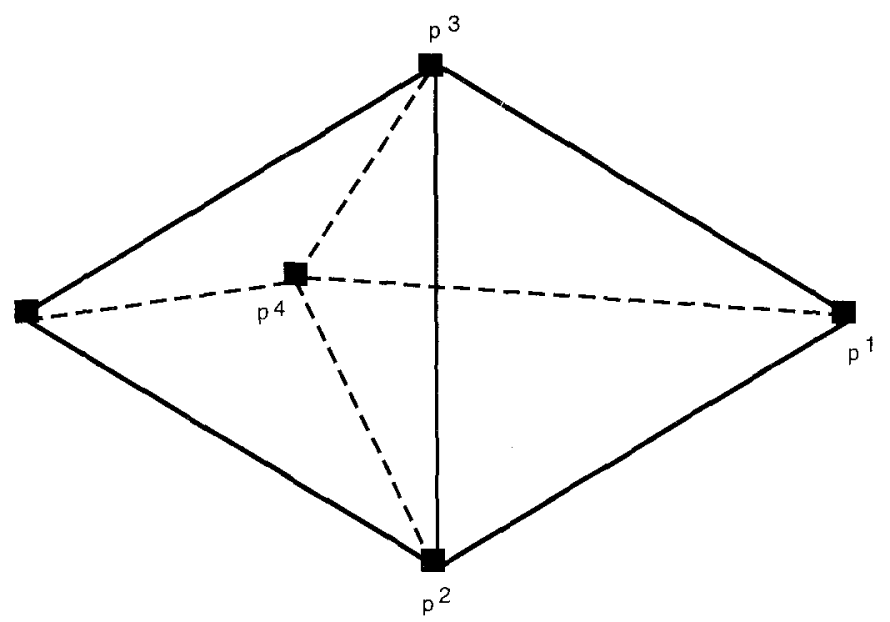

Fig. 1. Polytope with five extreme points in $\mathbb{R}^{3}$. The lines are edges. $\left\langle p^{1}, p^{2}, p^{3}, p^{4}\right\rangle$ is a segment of order 1 of this polytope.

points of polyhedra associated with degenerate network linear programs. However, there is no known algorithm which enumerates all extreme points of a convex polytope defined by a general degenerate system of linear constraints in time polynomial in the size of input and output. Although we are unable to present such an algorithm, we present an algorithm for enumerating all faces in time polynomial in their number and the size of input. For this task, we define a new concept, that of segments of a convex polytope. Given a convex polytope $\boldsymbol{\Gamma}$ of dimension $n$, for $1 \leqslant s \leqslant n-2$, a segment of $\boldsymbol{\Gamma}$ of order $s$ is the convex hull $\boldsymbol{\Omega}$ of a subset of extreme points of $\boldsymbol{\Gamma}$ which has the same dimension as $\boldsymbol{\Gamma}$, and satisfies the property that for all $1 \leqslant t \leqslant s$, every face of $\boldsymbol{\Omega}$ of dimension $t$ is a face of $\boldsymbol{\Gamma}$. In a general step, starting from a segment $\boldsymbol{\Omega}$ of order $s$ of the original polytope $\Gamma$ of dimension $n$, if $s<n-1$, the proposed algorithm tries to find a segment of order $s+1$ by checking whether there is an $(s+1)$-face of $\boldsymbol{\Omega}$ which is not a face of $\boldsymbol{\Gamma}$. If such an $(s+1)$-face is found, the algorithm is guaranteed to find an extreme point of $\boldsymbol{\Gamma}$ that is not in $\boldsymbol{\Omega}$; this is included in the list of known extreme points of $\boldsymbol{\Gamma}$; the algorithm then builds up this list until its convex hull becomes a segment of $\boldsymbol{\Gamma}$ of order $s+1$.

\section{Some preliminaries}

If the minimum and maximum values of a variable $x_{j}$ over $x \in K$ are the same, $\alpha$ say, then $\boldsymbol{K}$ lies on the hyperplane $x_{j}=\alpha$ in $\mathbb{R}^{n}$. In this case fix $x_{j}$ at $\alpha$ in (1) and eliminate it from the system. Each extreme point of the reduced system becomes an extreme point of the original system when we include $x_{j}$ at value $\alpha$ in it. So, in the sequel we assume that the maximum value of any variable in (1) is strictly greater than its minimum value. The operations carried out here do not change the dimension of the set of feasible solutions of (1), which we continue to denote by $\boldsymbol{K}$. 
Let $x_{\mathrm{B}}$ be a basic vector for $(1)$, say $\left(x_{1}, \ldots, x_{m}\right)$ to be specific. $x_{\mathrm{N}}=\left(x_{m+1}, \ldots, x_{n}\right)$ is the nonbasic vector when considering this basic vector. Let $(B, N)$ be the partition of $A$ corresponding to the basic, nonbasic partition of $x$ into $\left(x_{\mathrm{B}}, x_{\mathrm{N}}\right)$. The equality constraints in (1) are equivalent to $x_{\mathrm{B}}=B^{-1} b-B^{-1} N x_{N}$. Using these, the basic variables $x_{\mathrm{B}}$ can be eliminated and system (1) expressed purely in terms of the nonbasic variables $x_{\mathrm{N}}$ as

$$
-B^{-1} N x_{\mathrm{N}}+B^{-1} b \geqslant 0, \quad x_{\mathrm{N}} \geqslant 0 .
$$

In (2) all the constraints are inequality constraints, and if $\bar{x}_{\mathrm{N}}$ is an extreme point solution for it, then $\left(\bar{x}_{\mathrm{B}}, \bar{x}_{\mathrm{N}}\right.$ ) (where $\bar{x}_{\mathrm{B}}=B^{-1} b-B^{-1} N \bar{x}_{\mathrm{N}}$ ) is an extreme point of (1), and vice versa. Thus enumerating extreme points of (1), or those of (2), form the same problem. In fact, the set of feasible solutions of (2) is $K$ itself, expressed in the space of nonbasic variables $x_{\mathrm{N}}$; in this space, $\boldsymbol{K}$ is a full-dimensional convex polytope. We will find it convenient to use this transformation.

Two extreme points of a convex polytope are said to be adjacent iff the line segment joining them is an edge. By Definition 1.1 we have the following characterization of adjacency of extreme points on the convex hull of a given set of points.

Characterization 2.1. Let $K_{2}=\left\langle p^{1}, \ldots, p^{t}\right\rangle \subset \mathbb{R}^{n}$, where each $p^{k}$ is an extreme point of $\boldsymbol{K}_{2}$ and all the points are distinct, with $t \geqslant 3$ and $n \geqslant 2$. Two extreme points of $\boldsymbol{K}_{2}$, $p^{1}$ and $p^{2}$ say, are adjacent on $K_{2}$ iff there exists a $c=\left(c_{1}, \ldots, c_{n}\right)$ satisfying (3). Solving (3) for $c$ can be posed as an LP using theorems of alternatives for systems of linear constraints:

$$
c\left(p^{1}-p^{2}\right)=0, \quad c\left(p^{1}-p^{k}\right)>0, \quad \text { for } k=3, \ldots, t .
$$

Definition 2.2 (Adjacency of edges). A pair of edges $e_{1}, e_{2}$ of a convex polytope are said to be adjacent on it if they have a common vertex, and if both of them together lie on a two-dimensional face of that polytope.

We now state the condition for checking whether two edges of $\boldsymbol{K}_{2}$ are adjacent, based on Definitions 2.2 and 1.1 , in a form similar to (3) that can be verified by solving a single LP, in the following proposition.

Proposition 2.3. Let $\boldsymbol{K}_{2}=\left\langle p^{1}, \ldots, p^{t}\right\rangle \subset \mathbb{R}^{n}$, where each $p^{k}$ is an extreme point of $\boldsymbol{K}_{2}$ and all the points are distinct. Suppose $e_{1}$, the line segment joining $p^{1}$ and $p^{2}$, and $e_{2}$, the line segment joining $p^{1}$ and $p^{3}$, are two edges of $\boldsymbol{K}_{2}$ with a common extreme point $p^{1}$. From Definitions 1.1 and $2.2, e_{1}, e_{2}$ are adjacent edges on $\boldsymbol{K}_{2}$ iff there exists a row vector $c \in \mathbb{R}^{n}, c \neq 0$, and a $\beta \in \mathbb{R}^{1}$, such that

$$
\begin{aligned}
& c p^{1}=c p^{2}=c p^{3}=\beta, \\
& c p^{t}<\beta, \quad \text { if } p^{t} \text { is an adjacent extreme point of } p^{1} \text { on } K_{2}, \\
& \quad \text { different from } p^{2} \text { and } p^{3}, \\
& c p^{k} \leqslant \beta, \quad \text { if } p^{k} \text { is not adjacent to } p^{1} \text { on } K_{2} .
\end{aligned}
$$


If the edges $e_{1}, e_{2}$ are adjacent on $\boldsymbol{K}_{2}$, the two-dimensional face of $\boldsymbol{K}_{2}$ containing them consists of all the extreme points $p^{k}$ of $\boldsymbol{K}_{2}$ satisfying $c p^{k}=\beta$, for $c, \beta$ satisfying (4).

Proof. By Definition 1.1, the edges $e_{1}, e_{2}$ form a two-dimensional face of $\boldsymbol{K}_{2}$ iff there exists a supporting hyperplane $\boldsymbol{H}$ for $\boldsymbol{K}_{2}$, determined by $c y=\beta$ say, which contains $e_{1}$ and $e_{2}$, and does not contain any other adjacent extreme point of $p^{1}$ on $K_{2}$. The system (4) is exactly a restatement of these conditions. When $e_{1}, e_{2}$ do form a two-dimensional face of $\boldsymbol{K}_{2}$, that face is the intersection of $\boldsymbol{K}_{2}$ with that hyperplane $\boldsymbol{H}$, and hence contains all the extreme points $p^{k}$ of $\boldsymbol{K}_{2}$ satisfying $c p^{k}=\beta$.

Let $K_{2}=\left\langle p^{1}, \ldots, p^{t}\right\rangle$ of dimension $n$ in $\mathbb{R}^{n}$ be such that each $p^{k}$ for $k=1, \ldots, t$ is an extreme point of $\boldsymbol{K}_{2}$. All the edges of $\boldsymbol{K}_{2}$ can be determined by using Characterization 2.1. Once the edges of $\boldsymbol{K}_{2}$ are determined, using them, the two-dimensional faces of $\boldsymbol{K}_{2}$ can be determined using the results in Proposition 2.3. This process can be continued to determine the $(s+1)$-dimensional faces of $\boldsymbol{K}_{2}$ efficiently from the $s$-dimensional faces and the edges of $\boldsymbol{K}_{2}$, using the results discussed in the following theorem, for all $s$.

Theorem 2.4, Let $\boldsymbol{K}_{2}=\left\langle p^{1}, \ldots, p^{t}\right\rangle$ of dimension $n$ in $\mathbb{R}^{n}$ be such that each $p^{k}$ for $k=1, \ldots, t$ is an extreme point of $\boldsymbol{K}_{2}$. For $n-1>s \geqslant 2$, every face $\boldsymbol{G}$ of $\boldsymbol{K}_{2}$ of dimension $s+1$ containing $p^{1}$ satisfies the following properties. Let $\Delta_{1}\left(\boldsymbol{K}_{2}\right), \Delta_{1}(\boldsymbol{G})$ be the adjacent vertices of $p^{1}$ on $\boldsymbol{K}_{2}$ and $\boldsymbol{G}$, respectively. Then there exists a face $\boldsymbol{F}$ of $\boldsymbol{K}_{2}$ of dimension $s$ containing $p^{1}$ such that if $\Delta_{1}(\boldsymbol{F})$ is the set of adjacent extreme points of $p^{1}$ on $\boldsymbol{F}$, then

(i) $\Delta_{1}(G)$ is a maximal subset of $\Delta_{1}\left(K_{2}\right)$ satisfying $\Delta_{1}(G) \supset \Delta_{1}(F)$, and the affine rank of $\left\{p^{1}\right\} \cup \Delta_{1}(\boldsymbol{G})$ is $s+1$;

(ii) there exists a $c \in \mathbb{R}^{n}, c \neq 0$, and a real number $\beta$ satisfying

$$
c p \begin{cases}=\beta, & \text { for all } p \in\left\{p^{1}\right\} \cup \Delta_{1}(\boldsymbol{G}), \\ <\beta, & \text { for all } p \in \Delta_{1}\left(\boldsymbol{K}_{2}\right) \backslash \Delta_{1}(\boldsymbol{G}), \\ \leqslant \beta, & \text { for all } p \in\left\{p^{1}, \ldots, p^{t}\right\} \backslash \Delta_{1}\left(\boldsymbol{K}_{2}\right) ;\end{cases}
$$

(iii) $\boldsymbol{G}=\boldsymbol{K}_{2} \cap \boldsymbol{H}$, where $\boldsymbol{H}$ is the hyperplane $\{x: c x=\beta\}$ where $c, \beta$ are from (ii). So, the extreme points of $\boldsymbol{G}$ are all the $p^{k}, k=1, \ldots, t$, which are on $\boldsymbol{H}$.

Conversely, if $\Delta_{1}(F)$ is the set of adjacent vertices of $p^{1}$ on an s-dimensional face of $\boldsymbol{K}_{2}$ containing $p^{1}$, and $\Delta_{1}(\boldsymbol{G})$ is a subset of $\Delta_{1}\left(\boldsymbol{K}_{2}\right)$ satisfying (i) and (ii), then $\boldsymbol{G}$ determined by (iii) is a face of $\boldsymbol{K}_{2}$ of dimension $s+1$.

Proof. By Definition 1.1, a face of a convex polytope is its intersection with a supporting hyperplane. So, if $\boldsymbol{G}$ is an $(s+1)$-dimensional face of $\boldsymbol{K}_{2}$, there must exist a $c \in \mathbb{R}^{n}$, $c \neq 0$, and a real number $\beta$, such that if $\boldsymbol{H}$ is the hyperplane $\{x: c x=\beta\}$, then $\boldsymbol{G}=\boldsymbol{K}_{2} \cap \boldsymbol{H}$ and $\boldsymbol{K}_{2} \subset\{x: c x \leqslant \beta\}$. So, if $\boldsymbol{G}$ is an $(s+1)$-dimensional face of $\boldsymbol{K}_{2}$ containing $p^{1}$, let $\boldsymbol{F}$ be an $s$-dimensional face of $\boldsymbol{G}$ containing $p^{1}$. Let $\Delta_{1}(\boldsymbol{G})$, $\Delta_{1}(\boldsymbol{F})$ be the sets of adjacent extreme points of $p^{1}$ on $\boldsymbol{G}$ and $\boldsymbol{F}$, respectively. So, the 
affine rank of $\left\{p^{1}\right\} \cup \Delta_{1}(F)$ is $s$, and the affine rank of $\left\{p^{1}, p\right\} \cup \Delta_{1}(F)$ is $s+1$ for any $p \in \Delta_{1}(\boldsymbol{G}) \backslash \Delta_{1}(\boldsymbol{F})$. When the set of edges joining $p^{1}$ to its adjacent vertices in $\{p\} \cup \Delta_{1}(\boldsymbol{F})$ determine an $(s+1)$-dimensional face of $\boldsymbol{K}_{2}$, this face $\boldsymbol{G}$ will contain all the points in $K_{2}$ in the affine hull of $\left\{p^{1}, p\right\} \cup \Delta_{1}(F)$, and hence it satisfies (i). So, (i) -(iii) hold.

Conversely, if $\Delta_{1}(\boldsymbol{G})$ is a set satisfying (i) and (ii), then the hyperplane $\boldsymbol{H}$ defined in (iii) is a supporting hyperplane for $\boldsymbol{K}_{2}$, and $\boldsymbol{G}=\boldsymbol{K}_{2} \cap \boldsymbol{H}$ is therefore a face of $\boldsymbol{K}_{2}$ and by (i) and (ii) its dimension is $s+1$.

Consider the convex polytope $\boldsymbol{K}$ defined by (1). The following is a characterization, standard in linear programming literature, of the dimension of the smallest dimension face of $\boldsymbol{K}$ containing a given set of edges of $\boldsymbol{K}$ with a common vertex.

Characterization 2.5. Let $p^{0}$ be an extreme point of the convex polytope $K$ defined by (1), and let $\left\{e_{1}, \ldots, e_{r}\right\}$ be a set of edges of $K$ incident at $p^{0}$. Let $p^{j}$ be the other extreme point of $K$ on $e_{j}$ for $j=1, \ldots, r$, and $s$ is the affine rank of $\left\{p^{0}, p^{1}, \ldots, p^{r}\right\}$. The dimension of the smallest dimension face of $\boldsymbol{K}$ containing all these edges $e_{1}, \ldots, e_{r}$ is $d$, the cardinality of $\left\{A_{. j}: j\right.$ such that $x_{j}>0$ in at least one of the points in $\left\{p^{0}, p^{1}, \ldots\right.$, $\left.\left.p^{r}\right\}\right\}$ minus the rank of the same set. The smallest dimension face of $\boldsymbol{K}$ containing all the edges $e_{1}, \ldots, e_{r}$ is unique, and its dimension $d$ is greater than or equal to $s$. If $\boldsymbol{F}$ is this face, it is possible that $\boldsymbol{F}$ contains some other edges incident at $p^{0}$ other than $e_{1}, \ldots, e_{r}$.

\section{Enumeration of facets of the convex hull of a given set of points}

Let $\left\{p^{1}, \ldots, p^{t}\right\}$ be a given set of points in $\mathbb{R}^{n}$ and let $\boldsymbol{P}=\left\langle p^{1}, \ldots, p^{t}\right\rangle$ be the convex hull of $\left\{p^{1}, \ldots, p^{t}\right\}$. Let $\operatorname{rank}\left\{p^{2}-p^{1}, \ldots, p^{t}-p^{1}\right\}=s$. Then $\boldsymbol{P}$ has dimension $s$. If $s=n, \boldsymbol{P}$ is a full-dimensional convex polytope in $\mathbb{R}^{n}$. If $s<n$, a system of $n-s$ equations characterizing the affine space of $\boldsymbol{P}$ can be determined, and by eliminating $n-s$ variables using it, one goes into the affine space of $\boldsymbol{P}$ in which $\boldsymbol{P}$ is a fulldimensional convex polytope. In this reduced system the facets of $\boldsymbol{P}$ can be determined by the procedure discussed below. Each facet leads to a linear inequality constraint for characterizing $\boldsymbol{P}$ through a system of linear constraints. From these and from the system of equations characterizing the affine space of $\boldsymbol{P}$ in $\mathbb{R}^{n}$, we get a linear constraint representation of $\boldsymbol{P}$.

So, we assume without any loss of generality that $\boldsymbol{P}$ is a full-dimensional convex polytope in $\mathbb{R}^{n}$. Let $\bar{p}=\left(p^{1}+\cdots+p^{t}\right) / t$. The point $\bar{p}$ is an interior point of $\boldsymbol{P}$. Let $\boldsymbol{Q}$ be the polytope obtained from $\boldsymbol{P}$ by translating the origin to $\bar{p}$, i.e., $\boldsymbol{Q}=\left\langle q^{1}, \ldots, q^{t}\right\rangle$ where $q^{k}=p^{k}-\bar{p}$, for $k=1, \ldots, t$. Hence, 0 is an interior point of $Q$, and thus is not contained on any of the facetal hyperplanes of $Q$. Thus every facetal hyperplane of $Q$ can be represented by an equation of the form $a_{1} x_{1}+\cdots+a_{n} x_{n}=1$ with $Q$ lying in the halfspace represented by $a_{1} x_{1}+\cdots+a_{n} x_{n} \leqslant 1$ (since $0 \in \boldsymbol{Q}$ ). We will represent this 
facetal hyperplane by the vector of coefficients $\left(a_{1}, \ldots, a_{n}\right)$. Then $a=\left(a_{1}, \ldots, a_{n}\right)$ is a vector representing a facetal hyperplane of $Q$, iff $a$ is an extreme point of the system of constraints

$$
a q^{k} \leqslant 1, \quad k=1, \ldots, t,
$$

in which $a$ is the vector of variables, and $q^{1}, \ldots, q^{t}$ are the data.

And, $a_{1} x_{1}+\cdots+a_{n} x_{n}=1$ represents a facetal hyperplane of $Q$ with $Q$ lying in the halfspace defined by $a_{1} x_{1}+\cdots+a_{n} x_{n} \leqslant 1$, iff $a_{1} x_{1}+\cdots+a_{n} x_{n}=1+a \bar{p}$ is a facetal hyperplane of $\boldsymbol{P}$ with $\boldsymbol{P}$ lying in the halfspace defined by $a_{1} x_{1}+\cdots+a_{n} x_{n} \leqslant 1+a \bar{p}$. Therefore, any algorithm for enumerating the extreme points corresponding to a system of linear constraints can be used to enumerate the facets of the convex hull of a given set of points.

Let $G=\{a: a$ is feasible to (6) $\}$ be the polytope in the space of the variables $a$. $G$ is known as the dual of the polytope $Q$. In the pair of polytopes $Q, G$, each extreme point of one corresponds to a facet of the other and vice versa.

\section{Segments of a polytope}

Definition 4.1 (Segment of order $s$ ). Let $\boldsymbol{\Omega}$ be the convex hull of a subset of extreme points of a convex polytope $\boldsymbol{\Gamma}$ of dimension $n$. For $1 \leqslant s \leqslant n-2, \Omega$ is called a segment of order $s$ of $\boldsymbol{\Gamma}$ if $\boldsymbol{\Omega}$ has the same dimension $n$ as $\boldsymbol{\Gamma}$, and every face of $\boldsymbol{\Omega}$ of dimension $t$ is also a face of $\boldsymbol{\Gamma}$ for all $1 \leqslant t \leqslant s$.

Thus, if $\boldsymbol{\Omega}$ is the convex hull of a subset of extreme points of a convex polytope $\Gamma$, $\boldsymbol{\Omega}$ is a segment of $\boldsymbol{\Gamma}$ of order 1 if $\boldsymbol{\Omega}$ has the same dimension as $\boldsymbol{\Gamma}$, and adjacency of extreme points on $\boldsymbol{\Omega}$ coincides with that on $\boldsymbol{\Gamma}$.

As an example, consider the convex polytope $\boldsymbol{\Gamma}_{3}$ of full dimension in $\mathbb{R}^{3}$ shown in Fig. 1, with five extreme points. $\boldsymbol{\Omega}_{3}$, the convex hull of $\left\{p^{1}, p^{2}, p^{3}, p^{4}\right\}$, is a segment of order 1 of $\boldsymbol{\Gamma}_{3}$, because it satisfies both the necessary conditions for being one. And $\Omega_{3}$ is not a segment of order 2 of $\boldsymbol{\Gamma}_{3}$, because $\left\langle p^{2}, p^{3}, p^{4}\right\rangle$ is a two-dimensional face of $\boldsymbol{\Omega}_{3}$ but not of $\boldsymbol{\Gamma}_{3}$.

As discussed earlier, in a simple (i.e., nondegenerate) polytope, the number of edges incident at any vertex is always equal to the dimension of the polytope. Hence the dimension condition implies that the only possible segment of a simple polytope is the whole polytope itself. However, a nonsimple polytope may have a segment which is a proper subset of it. Fig. 1 provides an example of this.

The polytope in Fig. 1 in $\mathbb{R}^{3}$ is a bipyramid that is the union of two three-dimensional simplices with a common base and disjoint interiors. Consider a bipyramid $\boldsymbol{\Gamma}^{n}$ in $\mathbb{R}^{n}$ that is the union of two $n$-dimensional simplices with a common base and disjoint interiors, and let $\boldsymbol{\Omega}^{n}$ be one of the two simplices (this will be a direct generalization of the bipyramid in Fig. 1 to $\mathbb{R}^{n}$ ). Clearly $\boldsymbol{\Omega}^{n}$ is a segment of order 1 of $\boldsymbol{\Gamma}^{n}$ for all $n \geqslant 3$. Furthermore, every face of $\boldsymbol{\Omega}^{n}$ of dimension smaller than or equal to $n-2$ is also a 


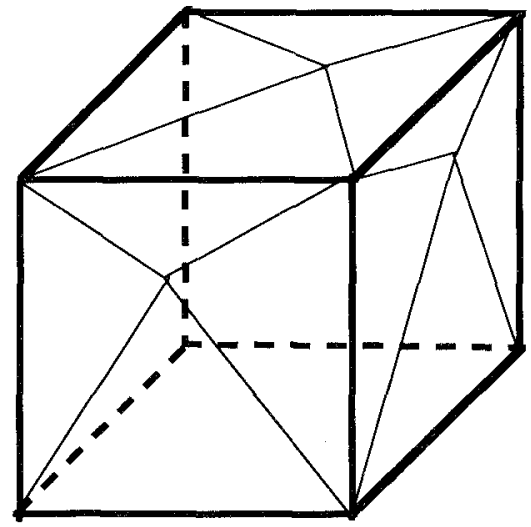

Fig. 2. $\boldsymbol{\Omega}_{1}$ is the unit cube in $\mathbb{R}^{3}$ with thick edges. $\boldsymbol{\Gamma}_{1}$ is the convex polytope containing $\boldsymbol{\Omega}_{1}$ as a segment of order 1 , plus six new extreme points (only three visible in the figure) with one on the outer side of each facet of $\Omega_{1}$.

face of $\Gamma^{n}$. So, $\boldsymbol{\Omega}^{n}$ is a segment of order $n-2$ of $\boldsymbol{\Gamma}^{n}$ for all $n \geqslant 4$. This example is due to [11].

Let $\boldsymbol{\Omega}$ be a segment of order 1 of a full-dimensional convex polytope $\boldsymbol{\Gamma}$ in $\mathbb{R}^{n}$. It is possible that none of the facetal hyperplanes of $\boldsymbol{\Gamma}$ is a facetal hyperplane of $\boldsymbol{\Omega}$. As an example, let $\boldsymbol{\Omega}_{1}$ be the unit cube in $\mathbb{R}^{3}$. Draw the normal to each facet of $\Omega_{1}$ through the center of that facet, and take a point on it a little bit outside of $\boldsymbol{\Omega}_{1}$ as a new extreme point. This generates a convex polytope $\Gamma_{1}$ in $\mathbb{R}^{3}$ with fourteen extreme points (eight extreme points of $\Omega_{1}$ and one extreme point on each of the six normal lines to the facets of $\Omega_{1}$ ). It can be verified that $\boldsymbol{\Omega}_{1}$ (outlined with thick edges in Fig. 2) is a segment of order 1 of $\boldsymbol{\Gamma}_{1}$. Each facet of $\boldsymbol{\Gamma}_{1}$ is a two-dimensional simplex, but none of the facetal hyperplanes of $\boldsymbol{\Gamma}_{1}$ is a facetal hyperplane of $\boldsymbol{\Omega}_{1}$.

There can exist full-dimensional convex polytopes $\boldsymbol{\Gamma}$ in $\mathbb{R}^{n}$ and segments $\boldsymbol{\Omega}$ of order 1 of $\boldsymbol{\Gamma}$ such that for every face $\boldsymbol{F}$ of $\boldsymbol{\Gamma}$ of dimension $r, 3 \leqslant r \leqslant n-1$, either $\boldsymbol{F} \cap \boldsymbol{\Omega}=\boldsymbol{F}$, or $\boldsymbol{F} \cap \boldsymbol{\Omega}$ has dimension $\leqslant r-1$. To construct an example like this, repeat the construction in Fig. 2, replacing the unit cube with any full-dimensional convex polytope $\boldsymbol{\Omega}_{2}$ in $\mathbb{R}^{n}$. That is, draw the normal to each facetal hyperplane of $\Omega_{2}$ through the center (or any relative interior point) of the corresponding facet, and select a point on this normal just outside $\boldsymbol{\Omega}_{2}$ as a new extreme point. So, the number of new extreme points added is equal to the number of facets of $\boldsymbol{\Omega}_{2}$. Let $\boldsymbol{\Gamma}_{2}$ be the convex hull of the union of $\boldsymbol{\Omega}_{2}$ and all these new extreme points. It can be verified that none of the facetal hyperplanes of $\Gamma_{2}$ is a facetal hyperplane of $\boldsymbol{\Omega}_{2}$, and that $\boldsymbol{\Omega}_{2}$ is a segment of order 1 of $\boldsymbol{\Gamma}_{2}$ which satisfies the properties mentioned above.

The propositions given below establish some simple properties of segments which may prove useful in the study of segments, but they may not all be used in the algorithm developed later on.

Proposition 4.2. Let $\boldsymbol{\Omega}$ be a segment of order 1 of a full-dimensional convex polytope $\boldsymbol{\Gamma}$ in $\mathbb{R}^{n}, \boldsymbol{\Omega} \neq \boldsymbol{\Gamma}$. If $\boldsymbol{\Gamma} \backslash \boldsymbol{\Omega}$ is a convex set, then $\boldsymbol{\Omega}_{1}$, the closure of $\boldsymbol{\Gamma} \backslash \Omega$, is a segment of 
order 1 of $\boldsymbol{\Gamma}$, and $\boldsymbol{\Omega} \cap \boldsymbol{\Omega}_{1}$ is a facet of $\boldsymbol{\Omega}$.

Proof. Since $\boldsymbol{\Omega}$ is a closed subset of $\boldsymbol{\Gamma}$ of the same dimension as $\boldsymbol{\Gamma}$ and $\boldsymbol{\Gamma} \backslash \boldsymbol{\Omega}$ is convex, $\boldsymbol{\Gamma} \backslash \boldsymbol{\Omega}$ has the same dimension as $\boldsymbol{\Gamma}$. So, $\boldsymbol{\Omega}_{1}$ has the same dimension as $\boldsymbol{\Gamma}$.

Since $\boldsymbol{\Gamma} \backslash \boldsymbol{\Omega}$ and $\boldsymbol{\Omega}$ are disjoint convex sets, they can be separated by a hyperplane. This hyperplane contains $\boldsymbol{\Omega} \cap \boldsymbol{\Omega}_{1}$, and hence $\boldsymbol{\Omega} \cap \boldsymbol{\Omega}_{1}$ is a face of both $\boldsymbol{\Omega}$ and $\boldsymbol{\Omega}_{1}$. Two convex polytopes of full dimension with a common face and with disjoint interiors form a union which is also convex, only if that common face is a common facet. So, $\boldsymbol{\Omega} \cap \boldsymbol{\Omega}_{1}$ is a common facet of both $\boldsymbol{\Omega}$ and $\boldsymbol{\Omega}_{1}$.

If $x^{1}$ and $x^{2}$ are two extreme points of $\boldsymbol{\Omega}_{1}$ at least one of which is not in $\boldsymbol{\Omega}$, then $x^{1}$ and $x^{2}$ are adjacent on $\boldsymbol{\Omega}_{1}$ iff they are adjacent on $\boldsymbol{\Gamma}$. And two extreme points of $\boldsymbol{\Omega} \cap \boldsymbol{\Omega}_{1}$ are adjacent on $\boldsymbol{\Omega}$ (and hence on $\boldsymbol{\Omega}_{1}$ ) iff they are adjacent on $\boldsymbol{\Gamma}$. These facts imply that $\boldsymbol{\Omega}_{1}$ is also a segment of order 1 of $\boldsymbol{\Gamma}$.

Proposition 4.3. Let $\boldsymbol{\Omega}$ be a segment of order 1 of a full-dimensional convex polytope $\boldsymbol{\Gamma}$ in $\mathbb{R}^{n}, \boldsymbol{\Omega} \neq \boldsymbol{\Gamma}$. If $\boldsymbol{\Gamma} \backslash \boldsymbol{\Omega}$ is the union of convex sets $\Delta_{1}, \ldots, \Delta_{r}$, where each $\Delta_{t}$ for $t=1, \ldots, r$ is a maximal convex set in the union, let $\bar{\Delta}_{t}$ be the closure of $\Delta_{t}$. Then $\bar{\Delta}_{t}$ is a segment of order 1 of $\boldsymbol{\Gamma}$, and $\bar{\Delta}_{t} \cap \boldsymbol{\Omega}$ is a facet of both $\boldsymbol{\Omega}$ and $\bar{\Delta}_{t}$, for each $t=1, \ldots, r$.

Proof. Since $\Delta_{t}$ is a maximal convex subset of $\boldsymbol{\Gamma} \backslash \boldsymbol{\Omega}$, it is clear that $\boldsymbol{\Gamma} \backslash \Delta_{t}$ is convex, and as in the proof of Proposition 4.2 it can be verified that $\Delta_{t}$ is full-dimensional.

Since both $\Delta_{t}$ and $\Gamma \backslash \Delta_{t}$ are convex and have disjoint interiors, they can be separated by a hyperplane. So, as in the proof of Proposition 4.2, it can be concluded that $\bar{\Delta}_{t} \cap \boldsymbol{\Omega}$ is a common facet of both of them, for $t=1, \ldots, r$.

If $x^{1}$ is an extreme point of $\boldsymbol{\Gamma}$ in $\Delta_{t}$ and $x^{2}$ is an extreme point of $\boldsymbol{\Gamma}$ in $\bar{\Delta}_{t}$, then the line segment $\left\{x: x=\alpha x^{1}+(1-\alpha) x^{2}, 0<\alpha \leqslant 1\right\} \subset \Delta_{t}$, and hence $x^{1}$ and $x^{2}$ are adjacent on $\bar{\Delta}_{t}$ iff they are adjacent on $\Gamma$, for $t=1, \ldots, r$. Also, two extreme points in $\bar{\Delta}_{t} \cap \boldsymbol{\Omega}$ are adjacent in $\bar{\Delta}_{t}$ iff they are adjacent in $\boldsymbol{\Gamma}$ since $\boldsymbol{\Omega}$ is a segment of order 1 of $\boldsymbol{\Gamma}$. So, $\bar{\Delta}_{t}$ is a segment of order 1 of $\boldsymbol{\Gamma}$ for $t=1, \ldots, r$.

Proposition 4.4. Let $\boldsymbol{\Omega}$ be a segment of order 1 of a full-dimensional convex polytope $\boldsymbol{\Gamma}$ in $\mathbb{R}^{n}$. If a two-dimensional face $\boldsymbol{T}$ of $\boldsymbol{\Gamma}$ has an intersection with $\boldsymbol{\Omega}$ that is more than an edge, then $\boldsymbol{T}$ is entirely contained in $\boldsymbol{\Omega}$.

Proof. Suppose $\boldsymbol{\Omega}$ has an intersection with $\boldsymbol{T}$ that is more than an edge, but does not contain all of $\boldsymbol{T}$. Then one of the facets of $\Omega$ splits $T$ through its relative interior, and the intersection of $\boldsymbol{T}$ and that facet must lead to an edge of $\boldsymbol{\Omega}$ in the facet; that edge is not an edge of $\boldsymbol{T}$, contradicting the segment property of $\boldsymbol{\Omega}$.

Proposition 4.5. Let $\boldsymbol{\Gamma}$ be a full-dimensional convex polytope in $\mathbb{R}^{3}$, and $\boldsymbol{\Omega} \neq \boldsymbol{\Gamma}$ a segment of order 1 of $\boldsymbol{\Gamma}$. Then there exists at least one facet $\boldsymbol{F}$ of $\boldsymbol{\Gamma}$ such that $\boldsymbol{F} \cap \boldsymbol{\Omega}$ has dimension smaller than or equal to 1 (i.e., $\boldsymbol{F} \cap \boldsymbol{\Omega}$ is either empty or $\boldsymbol{\Omega}$ contains at most an edge joining a pair of adjacent vertices on $\boldsymbol{F}$ ). 
Proof. Since $\boldsymbol{\Omega} \neq \boldsymbol{\Gamma}$, there must exist at least one facet of $\boldsymbol{\Gamma}, \boldsymbol{F}$ say, such that $\boldsymbol{\Omega}$ does not contain all of $\boldsymbol{F}$. Since $\boldsymbol{F}$ is three-dimensional and $\boldsymbol{F}$ is a two-dimensional face of $\boldsymbol{\Gamma}$, Proposition 4.4 implies that either $\boldsymbol{F} \cap \boldsymbol{\Omega}=\emptyset$, or $\boldsymbol{\Omega}$ contains at most an edge joining a pair of adjacent vertices on $F$.

Proposition 4.6. Let $\boldsymbol{\Omega}$ be a segment of a full-dimensional polytope $\boldsymbol{\Gamma}$ in $\mathbb{R}^{n}, n \geqslant 3$. If $\boldsymbol{\Omega} \neq \boldsymbol{\Gamma}$, there exists at least one face $\boldsymbol{F}$ of $\boldsymbol{\Gamma}$ of dimension greater than or equal to 2 , such that the dimension of $\boldsymbol{F} \cap \Omega$ is smaller than or equal to the dimension of $\boldsymbol{F}$ minus 1.

Proof. Proposition 4.5 implies that the theorem holds when $n=3$. So, consider $n \geqslant 4$. Now set up an induction hypothesis that the result in the proposition holds for every convex polytope $K_{3}$ of dimension $\leqslant n-1$ and a segment of it which is not the whole polytope $\boldsymbol{K}_{3}$. Under this induction hypothesis, we will now prove that the result in the proposition holds for the polytope $\boldsymbol{\Gamma}$ of dimension $n$ and its segment $\boldsymbol{\Omega} \neq \boldsymbol{\Gamma}$.

Since $\boldsymbol{\Omega} \neq \boldsymbol{\Gamma}$, there must exist a facet of $\boldsymbol{\Gamma}, \boldsymbol{G}$ say, such that $\boldsymbol{\Omega} \cap \boldsymbol{G} \neq \boldsymbol{G}$. If the dimension of $\boldsymbol{\Omega} \cap \boldsymbol{G}$ is the dimension of $\boldsymbol{G}$ minus 1, we are done since the facet $\boldsymbol{G}$ satisfies the property of the required face in the proposition. If $\boldsymbol{\Omega} \cap \boldsymbol{G}$ has the same dimension as $\boldsymbol{G}$, it is a segment of $\boldsymbol{G}$. By the induction hypothesis there exists a face $\boldsymbol{F}$ of $\boldsymbol{G}$ of dimension $\geqslant 2$ such that the dimension of $\boldsymbol{F} \cap \boldsymbol{\Omega}$ is smaller than or equal to the dimension of $\boldsymbol{F}$ minus 1 . Since $\boldsymbol{F}$ is also a face of $\boldsymbol{\Gamma}$, this shows that $\boldsymbol{F}$ satisfies the property stated in the proposition for $\boldsymbol{\Omega}, \boldsymbol{\Gamma}$. So, the result in the proposition also holds for the $n$-dimensional polytope $\boldsymbol{\Gamma}$ and its segment $\boldsymbol{\Omega} \neq \boldsymbol{\Gamma}$ under the induction hypothesis. We already verified that the result in the proposition holds for polytopes of dimension 3. So, by induction, it holds in general.

Proposition 4.7. Let $\boldsymbol{\Gamma}$ be a convex polytope of dimension 3 , and $\boldsymbol{\Omega} \neq \boldsymbol{\Gamma}$ a segment of it. Then $\boldsymbol{\Omega}$ has to be of order 1, i.e., there exists a pair of edges of $\boldsymbol{\Omega}$ with a common vertex which are adjacent on $\boldsymbol{\Omega}$ but not on $\boldsymbol{\Gamma}$.

Proof. Since $\boldsymbol{\Omega} \neq \boldsymbol{\Gamma}$, there must exist a facet of $\boldsymbol{\Omega}, \boldsymbol{F}_{1}$ say, which is not a facet of $\boldsymbol{\Gamma}$. Let $x^{1}$ be a vertex on $\boldsymbol{F}_{1}$. Since $\boldsymbol{\Omega}$ has dimension $3, \boldsymbol{F}_{1}$ is a two-dimensional polytope. If $e_{1}, e_{2}$ are edges of $\boldsymbol{F}_{1}$ incident at $x^{1}$, these edges $e_{1}, e_{2}$ with a common vertex $x^{1}$ are adjacent on $\boldsymbol{\Omega}$, but not on $\boldsymbol{\Gamma}$ since $\boldsymbol{F}_{1}$ is not a facet of $\boldsymbol{\Gamma}$, proving the proposition.

Proposition 4.8. Let $\boldsymbol{\Gamma}$ be a convex polytope of dimension 4, and let $\boldsymbol{\Omega} \neq \boldsymbol{\Gamma}$ be a segment of it. Then either (i) there exists a facet $\boldsymbol{F}$ of $\boldsymbol{\Gamma}$ such that the dimension of $\boldsymbol{F} \cap \boldsymbol{\Omega}$ is smaller than or equal to the dimension of $\boldsymbol{F}$ minus 1 or (ii) $\boldsymbol{\Omega}$ is not a segment of order 2 of $\boldsymbol{\Gamma}$.

Proof. Suppose (i) does not hold. So, every facet of $\boldsymbol{\Gamma}$ has a three-dimensional intersection with $\boldsymbol{\Omega}$. Since $\boldsymbol{\Omega} \neq \boldsymbol{\Gamma}$, there must exist a facet of $\boldsymbol{\Gamma}, \boldsymbol{F}_{1}$ say, such that $\boldsymbol{F}_{1} \cap \boldsymbol{\Omega} \neq \boldsymbol{F}_{1}$. So, $\boldsymbol{F}_{1} \cap \boldsymbol{\Omega}$ is a facet of $\boldsymbol{\Omega}$, and is a segment of order 1 of $\boldsymbol{F}_{1}$. By Proposition 4.5, 
there exist a pair of edges of $\boldsymbol{F}_{1} \cap \Omega, e_{1}, e_{2}$ say, which have a common vertex and are adjacent on $\boldsymbol{F}_{1} \cap \boldsymbol{\Omega}$ but not adjacent on $\boldsymbol{F}_{1}$. So, $e_{1}, e_{2}$ are adjacent on $\boldsymbol{\Omega}$ but not on $\boldsymbol{\Gamma}$, proving that (ii) holds.

Proposition 4.9. Let $\boldsymbol{\Omega}$ be a segment of order $r$ of a convex polytope $\boldsymbol{\Gamma}$ of dimension n. Let $\boldsymbol{F}$ be a face of $\boldsymbol{\Gamma}$ of dimension $u \geqslant r+1$. If $\boldsymbol{\Omega} \cap \boldsymbol{F}$ has dimension $u$, then $\boldsymbol{\Omega} \cap \boldsymbol{F}$ is a segment of order $r$ of $\boldsymbol{F}$.

Proof. A face of a face of a polytope is also a face of the original polytope. And the intersection of two faces is a face. Using these facts, this result follows directly from the definitions.

Proposition 4.10. Let $\Omega=\left\langle p^{1}, \ldots, p^{t}\right\rangle$ be a segment of order $r \leqslant n-2$ of a convex polytope $\boldsymbol{\Gamma}$ of dimension $n$, such that $\boldsymbol{\Omega} \neq \boldsymbol{\Gamma}$. Then $\boldsymbol{\Omega}$ has a facet which is not a facet of $\boldsymbol{\Gamma}$.

Proof. By hypothesis, all the points in $\left\{p^{1}, \ldots, p^{t}\right\}$ are extreme points of $\boldsymbol{\Gamma}$, and there exists at least one extreme point of $\boldsymbol{\Gamma}$ which is not in $\boldsymbol{\Omega}$. If all the facets of $\boldsymbol{\Omega}$ are facets of $\boldsymbol{\Gamma}$, then $\boldsymbol{\Omega}$ would be $\boldsymbol{\Gamma}$, a contradiction. So, there must be at least one facet of $\boldsymbol{\Omega}$ which is not a facet of $\boldsymbol{\Gamma}$.

Theorem 4.11. Let $\boldsymbol{\Omega}=\left\langle p^{1}, \ldots, p^{t}\right\rangle$ be a segment of order $s, 1 \leqslant s \leqslant n-2$, of $\boldsymbol{K}$ defined by (1). Let $\boldsymbol{G}$ be an $(s+1)$-dimensional face of $\boldsymbol{\Omega}$, containing an extreme point, $p^{1}$ say, and let $\Delta_{1}(G)$ be the set of adjacent extreme points of $p^{1}$ on $\boldsymbol{G}$. Then $\boldsymbol{G}$ is a face of $K$ iff $\operatorname{rank}\left\{A_{. j}: j\right.$ such that $x_{j}>0$ in at least one of the extreme points in the set $\left\{p^{1}\right\}$ $\left.\cup \Delta_{1}(G)\right\}$ is equal to its cardinality $-(s+1)$.

Proof. Let $\boldsymbol{E}$ be the set of edges of $\boldsymbol{\Omega}$ (and hence of $\boldsymbol{K}$ ) obtained by joining $p^{1}$ to a vertex $p \in \Delta_{1}(\boldsymbol{G})$. By hypothesis, the set of edges $\boldsymbol{E}$ determines the $(s+1)$-dimensional face $\boldsymbol{G}$ of $\boldsymbol{\Omega}$. If the condition holds, $\boldsymbol{E}$ also determines an $(s+1)$-dimensional face, $\boldsymbol{F}$ say, of $\boldsymbol{K}$. Let $\boldsymbol{H}$ be a supporting hyperplane of $\boldsymbol{K}$ such that $\boldsymbol{F}=\boldsymbol{H} \cap \boldsymbol{K}$. Then $\boldsymbol{G}=\boldsymbol{H} \cap \boldsymbol{\Omega}$. So, $\boldsymbol{G} \subset \boldsymbol{F}$, and since $\boldsymbol{\Omega}$ is a segment of order $s$ of $\boldsymbol{K}, \boldsymbol{G}=\boldsymbol{\Omega} \cap \boldsymbol{F}$ is a segment of order $s$ of $\boldsymbol{F}$ by Proposition 4.9. Hence, all facets of $\boldsymbol{G}$ are facets of $\boldsymbol{F}$, and since $\boldsymbol{G} \subset \boldsymbol{F}$, this implies that $\boldsymbol{G}=\boldsymbol{F}$. So, $\boldsymbol{G}$ is an $(s+1)$-dimensional face of $\boldsymbol{K}$.

Suppose $\boldsymbol{\Omega}$ is the convex hull of a subset of extreme points of $\boldsymbol{K}$, the set of feasible solutions of (1), of full dimension. For $s \geqslant 1$, if $\Omega$ is a segment of $\boldsymbol{K}$ of order $s$, and all its $s$-dimensional faces are determined, then the results in Theorems 2.4 and 4.11 can be used to generate the $(s+1)$-dimensional faces of $\boldsymbol{\Omega}$, and to check whether in fact $\boldsymbol{\Omega}$ is a segment of $\boldsymbol{K}$ of order $s+1$. If $\boldsymbol{\Omega}$ is not a segment of order $s+1$ of $\boldsymbol{K}$, this procedure will determine a vector $c$, and number $\beta$ such that $\boldsymbol{\Omega} \subset\{x: c x \leqslant \beta\}$, and there exists at least one extreme point of $\boldsymbol{K}$ not in $\boldsymbol{\Omega}$ in the half-space $\{x: c x \geqslant \beta\}$. 


\section{Algorithm for enumerating extreme points and faces of $K$}

Consider the convex polytope $\boldsymbol{K}$ defined by (1). This algorithm is initiated with one extreme point of $\boldsymbol{K}$ obtained by solving, for example, a Phase I problem using any of the polynomially bounded algorithms for LP. Beginning with this, the algorithm develops a list of extreme points of $\boldsymbol{K}$, adding at least one new extreme point to the list per iteration, until all of them are in. The computation also produces faces of $\boldsymbol{K}$ of all dimensions.

At some stage suppose the list is $\left\{d^{1}, \ldots, d^{r}\right\}$, consisting of $r$ distinct extreme points of $\boldsymbol{K}$. Let $\boldsymbol{K}_{1}=\left\langle d^{1}, \ldots, d^{r}\right\rangle$. At this stage we need to check whether there is an extreme point of $\boldsymbol{K}$ which is not in $\boldsymbol{K}_{1}$. This involves checking: is $\boldsymbol{K} \subseteq \boldsymbol{K}_{1}$ ? If $\boldsymbol{K}_{1}$ is specified as the convex hull of a general set of rational points, this problem is known to be NP-complete [7]. However, in our problem $\boldsymbol{K}_{1}$ is a special set, it is the convex hull of a subset of extreme points of $\boldsymbol{K}$, and the complexity of this problem in this special case is not known. Most of the computation in the algorithm discussed below is concerned with answering questions of this type, but this process produces faces of higher dimensions of $\boldsymbol{K}$ as a by-product.

Let $\left(x_{\mathrm{B}}, x_{\mathrm{N}}\right)$ be a partition of the variables in (1) into basic and nonbasic parts for some basic vector for (1). This partition is never changed during the algorithm.

We will now discuss an algorithm for enumerating all the faces of $\boldsymbol{K}$ based on the results discussed in Sections 2 and 4. When we use the result in Proposition 2.3 to identify a two-dimensional face of the convex hull of the current subset of extreme points of $\boldsymbol{K}$, it yields all the extreme points of that face; the face itself can be stored by storing its edge graph (i.e., one-dimensional skeleton). Later on, when we use the result in Theorem 2.4 to find a face of dimension greater than 2 of the convex hull of a subset of extreme points of $\boldsymbol{K}$, it also yields all the extreme points of that face; again the face itself can be stored by storing its edge graph. All these faces can also be stored by storing the vector $c$ feasible to (4) or (5) corresponding to it; the face itself is the set of optimum solutions for the problem of maximizing $c x$ over $\boldsymbol{K}$.

\section{An algorithm for generating all faces of $K$.}

Initialization. Find an extreme point of $\boldsymbol{K}$ using a Phase I procedure. With it go to the first iteration.

General iteration. Suppose $\left\{d^{1}, \ldots, d^{r}\right\}$ is the present list of extreme points of $\boldsymbol{K}$.

Step 1. For $k=1, \ldots, r$, let $\left(d_{\mathrm{B}}^{k}, d_{\mathrm{N}}^{k}\right)$ be a partition of the vector $d^{k}$ into basic and nonbasic parts as in the partition $\left(x_{\mathrm{B}}, x_{\mathrm{N}}\right)$ of the variables in (1). Here we check whether the dimension of $\boldsymbol{K}_{1}=\left\langle d^{1}, \ldots, d^{r}\right\rangle$ is smaller than $n-m$, the dimension of $\boldsymbol{K}$. This step is carried out only if in the previous iteration this step resulted in the affirmative answer for the corresponding question at that stage, otherwise we go directly to Step 2 in this iteration.

The dimension of $\boldsymbol{K}_{1}$ is the rank of $\left\{d_{\mathrm{N}}^{k}-d_{\mathrm{N}}^{1}: k=1, \ldots, r\right\}$. If it is $n-m$, go to Step 2, and in all subsequent iterations omit this step. If it is smaller than or equal to $n-m-1$, there exists an $f_{\mathrm{N}}=\left(f_{m+1}, \ldots, f_{n}\right) \neq 0$ such that $f_{\mathrm{N}}\left(d_{\mathrm{N}}^{k}-d_{\mathrm{N}}^{1}\right)=0$ for all $k=2, \ldots, r$; find such a vector $f_{\mathrm{N}}$. Let $f_{\mathrm{N}} d_{\mathrm{N}}^{1}=\beta$. Then all the extreme points $d^{1}, \ldots, d^{r}$ 
in the current list correspond to points in the $x_{\mathrm{N}}$-space lying on the hyperplane defined by $f_{\mathrm{N}} x_{\mathrm{N}}=\beta$. Now solve the two LPs, minimize $f_{\mathrm{N}} x_{\mathrm{N}}$, and maximize $f_{\mathrm{N}} x_{\mathrm{N}}$ subject to (1). One or both of these LPs will have as an optimum extreme point a point not in the current list. Call it $d^{r+1}$, add it to the list and go to the next iteration.

Step 2. In this step the algorithm tries to find a pair of points in the current list $\left\{d^{1}, \ldots, d^{r}\right\}$ satisfying the property that they are not adjacent on $\boldsymbol{K}$, but adjacent on $\boldsymbol{K}_{1}=$ $\left\langle d^{1}, \ldots, d^{r}\right\rangle$. Adjacency of some pairs of extreme points of $\boldsymbol{K}_{1}$ may have already been determined in previous iterations. If $p=\left(p_{j}\right), q=\left(q_{j}\right)$ from $\left\langle d^{1}, \ldots, d^{r}\right\rangle$ have not been checked for adjacency earlier, we know that they are not adjacent on $\boldsymbol{K}$ iff the rank of the set of vectors $\left\{A_{. j}: j\right.$ such that at least one of $p_{j}$ or $q_{j}$ or both are greater than 0$\}$ is strictly less than its cardinality -1 . And $p, q$ are adjacent on $K_{1}$ iff the following system in variables $c=\left(c_{1}, \ldots, c_{n}\right)$ has a feasible solution, which can be checked by solving an LP:

$$
\begin{aligned}
& c(p-q)=0, \\
& c\left(p-d^{k}\right)>0, \quad \text { for all } k \text { such that } d^{k} \neq p \text { or } q .
\end{aligned}
$$

If some new edges of $\boldsymbol{K}_{1}$ are found which are also edges of $\boldsymbol{K}$, store them.

If a pair of extreme points $p, q$ in the current list adjacent on $\boldsymbol{K}_{1}$ but not on $\boldsymbol{K}$ is found, let $\bar{c}$ be the vector obtained as the feasible solution for (7) for that pair. Now solve the LP

$$
\begin{array}{ll}
\operatorname{maximize} & \bar{c} x \\
\text { subject to } & (1) .
\end{array}
$$

The maximum objective value in (8) will be greater than or equal to $\gamma=\bar{c} p=\bar{c} q>$ $\min \left\{\bar{c} d^{k}: k=1, \ldots, r\right.$ such that $d^{k} \neq p$ or $\left.q\right\}$. If the maximum objective value in (8) is greater than $\gamma$, an extreme point optimum for it is a new extreme point of $\boldsymbol{K}$; add it to the list and go to the next iteration beginning with Step 2 .

If the maximum objective value in (8) is $\gamma$, and an optimum extreme point obtained for it when (8) is solved is either $p$ or $q$, the set of optimum solutions for (8) is a face $\boldsymbol{S}$ of $\boldsymbol{K}$ determined by

$$
A x=b, \quad \bar{c} x=\gamma, \quad x \geqslant 0 .
$$

In this case, $p, q$ are the only two points from the current list feasible to (9) (this follows from (7) by the choice of $\bar{c}$ ). Hence, its set of feasible solutions, the face $S$ of $K$, contains only $p, q$ from the current list. Since $S$ contains $p, q$ which are not adjacent on $\boldsymbol{K}$ (and hence not adjacent on $\boldsymbol{S}$, since $\boldsymbol{S}$ is a face of $\boldsymbol{K}$ ), the dimension of $\boldsymbol{S}$ must be greater than or equal to 2 . So, $S$, the set of feasible solutions of (9), has dimension greater than or equal to 2 and we only have two extreme points $p, q$ on it in the current list. Therefore, by applying Step 1 discussed earlier to (9) with $p, q$ as the only known extreme points on it at this stage, we can get a new extreme point of $S$; this will be an extreme point of $\boldsymbol{K}$ since $\boldsymbol{S}$ is a face of $\boldsymbol{K}$, add it to the list and go to the next iteration beginning with Step 2. 
If there exist no pair of extreme points which are adjacent on $K_{1}$ but not on $K$ in the current list, then all the edges of $\boldsymbol{K}_{1}=\left\langle d^{1}, \ldots, d^{r}\right\rangle$ are edges of $\boldsymbol{K}$, so $\boldsymbol{K}_{1}$ is a segment of order 1 of $\boldsymbol{K}$; go to Step 3 .

Step 3. When we come to this step, $K_{1}=\left\langle d^{1}, \ldots, d^{r}\right\rangle$, the convex hull of the current list, is a segment of order 1 of $\boldsymbol{K}$.

All the edges of $\boldsymbol{K}_{1}$ would have been determined already at this stage. The adjacency of some pairs of edges of $\boldsymbol{K}_{1}$ may have already been determined in previous iterations. If a pair of edges of $\boldsymbol{K}_{1}$ with a common vertex has not been checked for adjacency earlier, we can check whether they are adjacent on $\boldsymbol{K}_{1}$ using the result in Proposition 2.3; and if they are adjacent on $\boldsymbol{K}_{1}$, we can check whether they are adjacent on $\boldsymbol{K}$ using Characterization 2.5. If some new two-dimensional faces of $K_{1}$ are found which are also two-dimensional faces of $\boldsymbol{K}$, store them. If every pair of adjacent edges of $\boldsymbol{K}_{1}$ is also adjacent on $\boldsymbol{K}$, all two-dimensional faces of $\boldsymbol{K}_{1}$ are also two-dimensional faces of $\boldsymbol{K}$; so $\boldsymbol{K}_{1}$ is a segment of order 2 of $\boldsymbol{K}$, go to Step 4.

If we find a pair of adjacent edges of $\boldsymbol{K}_{1}, e_{1}, e_{2}$ say, which are not adjacent on $\boldsymbol{K}$, let $d^{1}$ be the common vertex, and $d^{2}, d^{3}$ the other vertices on them. So, in this case we have a row vector $c$ in $\mathbb{R}^{n}$ and a $\beta \in \mathbb{R}^{1}$ satisfying

$$
\begin{aligned}
& c d^{1}=c d^{2}=c d^{3}=\beta, \\
& c d^{t}<\beta, \quad \text { if } d^{t} \text { is an adjacent extreme point of } d^{1} \text { on } \boldsymbol{K}_{1}, \\
& \quad \text { different from } d^{2} \text { and } d^{3}, \\
& c d^{k} \leqslant \beta, \quad \text { if } d^{k} \text { is not adjacent to } d^{1} \text { on } K_{1} .
\end{aligned}
$$

Now solve the LP

$\begin{array}{ll}\text { maximize } & c x \\ \text { subject to } & (1) .\end{array}$

The optimum objective value in (11) is greater than or equal to $\beta$. If it is greater than $\beta$, an extreme point optimum for (11) yields an extreme point of $\boldsymbol{K}$ not in $\boldsymbol{K}_{1}$, add it to the list and go to the next iteration beginning with Step 2 .

If the optimum objective value in (11) is $\beta$, and if the extreme point optimum obtained is not in $K_{1}$, then again we have a new extreme point, add it to the list and go to the next iteration beginning with Step 2. On the other hand, suppose an extreme point of $K_{1}$ is obtained as an optimum solution of (11). Now consider the LP

$$
\begin{array}{ll}
\operatorname{maximize} & c x \\
\text { subject to } & x \in \boldsymbol{K}_{1} .
\end{array}
$$

We know from these conditions that the optimum objective value in (12) is $\beta$. Among adjacent extreme points of $d^{1}$ on $K_{1}$, the only ones which are optimum to (12) are $d^{2}, d^{3}$. This implies that the set of optimum solutions for (12) is the two-dimensional face $\boldsymbol{F}$ say, of $\boldsymbol{K}_{1}$ determined by the edges $e_{1}, e_{2}$. However, since $e_{1}, e_{2}$ do not form a two-dimensional face of $K$, the set of optimum solutions for (11) has dimension at least 3 , and contains $F$. That set is the set of feasible solutions of

$$
A x=b, \quad c x=\beta, \quad x \geqslant 0 .
$$


Let $\Delta$ be the set of extreme points on $F$. The affine rank of $\Delta$ is 2 , and it is the subset of extreme points of (13) in the present list. By applying Step 1 to the system (13) and the current known set $\Delta$ of extreme points of it, we can get a new extreme point of the set of feasible solutions of (13). This set is the set of optimum solutions of (11), and hence it is a face of $\boldsymbol{K}$, so that new extreme point of (13) is a new extreme point of $K$; add it to the list and go to the next iteration beginning with Step 2.

Step 4. When we come to this step, $K_{1}=\left\langle d^{1}, \ldots, d^{r}\right\rangle$, the convex hull of the current list, is a segment of order 2 of $\boldsymbol{K}$. All the edges and two-dimensional faces of $K_{1}$ would have been determined already at this stage. Using them, Theorem 2.4, and Characterization 2.5, look for a three-dimensional face of $K_{1}$ which is not a threedimensional face of $K$. (If this is not the first pass through Step 4, some of the three-dimensional faces of $\boldsymbol{K}_{1}$ would already have been determined, all these are threedimensional faces of $\boldsymbol{K}$. It is only necessary to check three-dimensional faces of $\boldsymbol{K}_{1}$ that can be formed using two-dimensional faces and at least one new edge that has been formed since the last pass through Step 4.) If some new three-dimensional faces of $\boldsymbol{K}_{1}$ are found which are also three-dimensional faces of $\boldsymbol{K}$, store them.

If a three-dimensional face of $K_{1}$ that is not a three-dimensional face of $\boldsymbol{K}$ is found, using the vector $c$ feasible to the system of the form (5) for it, find an extreme point of $\boldsymbol{K}$ not in $\boldsymbol{K}_{1}$ as in Steps 2 and 3 (this needs maximizing $c x$ over $\boldsymbol{K}$, and possibly using Step 1 on the set of optimum solutions of this LP). Add the new extreme point to the list. Go to the next iteration beginning in Step 2, with the new list.

If all the three-dimensional faces of $K_{1}$ are also three-dimensional faces of $\boldsymbol{K}, K_{1}$ is a segment of $K$ of order 3, go to Step 5, obtained by setting $s=3$ in the following general step.

General step $s+2$. When we come to this step, $K_{1}=\left\langle d^{1}, \ldots, d^{r}\right\rangle$, the convex hull of the current list, is a segment of order $s$ of $\boldsymbol{K}$. All the faces of $\boldsymbol{K}_{1}$ of dimension smaller than or equal to $s$ would have been determined already at this stage. Using the results in Theorems 2.4 and 4.11, look for a new $(s+1)$-dimensional face of $\boldsymbol{K}_{1}$ that is not a face of $\boldsymbol{K}$; store all the new $(s+1)$-dimensional faces of $\boldsymbol{K}_{1}$ which are also faces of $\boldsymbol{K}$ found in this process. If one $(s+1)$-dimensional face of $\boldsymbol{K}_{1}$ which is not a face of $\boldsymbol{K}$ is found, using the vector $c$ feasible to the system of the form (5) for it, find an extreme point of $\boldsymbol{K}$ not in $\boldsymbol{K}_{1}$ as in Steps 2 and 3; add this new extreme point to the list, and with the new list go to the next iteration beginning with Step 2 .

On the other hand, if all the $(s+1)$-dimensional faces of $\boldsymbol{K}_{1}$ are also faces of $\boldsymbol{K}, \boldsymbol{K}_{\mathbf{1}}$ is a segment of order $s+1$ of $K$. If $s+1=n-1$, the set of all the faces of $K$ consists of all the faces in storage now, and $\emptyset$, and $K$, terminate the algorithm. If $s+1<n-1$, go to Step $s+3$.

\section{Discussion}

Whenever a face of $K_{1}$, the convex hull of the current list of extreme points, is obtained in this algorithm, it is either a face of $\boldsymbol{K}$, or from the vector $c$ found in the process of finding this face, a new extreme point of $K$ not in $K_{1}$ is found by solving 
at most three LPs. In this algorithm faces of dimension $s+1$ of $K_{1}$ are found by building up an $s$-dimensional face with newly found edges that enhance its dimension by 1 maximally. From this it can be verified that the time complexity of this algorithm is polynomial in the unknown number of faces of $\boldsymbol{K}$ and the size (i.e., the number of digits in the binary encoding of the data) of (1). Let $L$ be the size of the original system (1), $\ell_{0}$ be the total number of extreme points of $\boldsymbol{K}, \ell_{1}$ be the total number of edges of $\boldsymbol{K}$ and $\ell$ be the total number of nonempty faces of $\boldsymbol{K}$ of all dimensions. Then the size of any system of the form (4) or (5) whose feasibility needs to be checked during the algorithm is at most $\mathrm{O}\left(\ell_{0} n L\right)$. Hence each LP to be solved during the algorithm needs an effort of at most $\mathrm{O}\left(\ell_{0} L n^{3.5}\right)$. And the number of LPs to be solved is at most $O\left(\ell \ell_{1}\right)$. Since this is the major work during the algorithm, its overall complexity is at most $\mathrm{O}\left(\ell \ell_{1} \ell_{0} L n^{3.5}\right)$.

So, for the problem of enumerating all the faces of $\boldsymbol{K}$, this algorithm has complexity bounded above by a polynomial in their number.

However, for the problem of enumerating just the extreme points of $\boldsymbol{K}$, this algorithm may not be efficient since the total number of faces could grow exponentially in the number of extreme points. For developing an efficient algorithm to enumerate extreme points using this approach, we need an efficient technique for checking whether a given segment $\boldsymbol{K}_{1}$ of order $s$ of $\boldsymbol{K}$ for some small value of $s$ (like 1, 2 or 3) is equal to $\boldsymbol{K}$, or find an extreme point of $\boldsymbol{K}$ that is not in $\boldsymbol{K}_{\mathrm{t}}$.

This remains a challenging problem. What we need is an algorithm for this problem that either determines that $\boldsymbol{K}_{1}=\boldsymbol{K}$, or finds a facetal hyperplane $\{x: c x=\beta\}$ of $\boldsymbol{K}_{\mathbf{1}}$ that is not a facetal hyperplane of $\boldsymbol{K}$, in time polynomial in the size of (1) and $r$, the number of extreme points of $\boldsymbol{K}_{1}$. It is not known whether such an algorithm exists. For this we are studying the conjectures given below.

Let $\boldsymbol{\Gamma}$ be a polytope of dimension $n$ in $\mathbb{R}^{n}$ represented through a system of linear constraints, $D y \leqslant d$ say, where $D$ is a matrix of order $m_{1} \times n$, and $\Gamma_{1}=\left\langle\left\{p^{1}, \ldots, p^{t}\right\}\right\rangle$, the convex hull of a subset of extreme points of $\boldsymbol{\Gamma}$, which is a segment of order 1 of $\boldsymbol{\Gamma}$. Without any loss of generality assume that the origin 0 is in the interior of the segment $\boldsymbol{\Gamma}_{1}$; this can be achieved by finding an interior point of $\boldsymbol{\Gamma}_{1}$, say the average of its extreme points, and translating the origin to it if necessary. Since 0 is an interior point of $\boldsymbol{\Gamma}$, we have $d>0$, so, by scaling the rows of $D$ we can convert $d$ into the vector of all ones. So, $\boldsymbol{\Gamma}$ is the set of feasible solutions of a system of the form

$$
D_{i .} y \leqslant 1, \quad i=1, \ldots, m_{1} .
$$

Each facet of $\Gamma$ is the set of feasible solutions of (14) satisfying a specified constraint in (14) as an equation. We assume that none of the constraints in (14) is a redundant constraint. Then, for each $i=1, \ldots, m_{1}$, the system

$$
D_{i} \cdot y=1, \quad D_{u} \cdot y \leqslant 1, \quad \text { for } u=1, \ldots, m_{1}, u \neq i,
$$

defines a facet of $\boldsymbol{\Gamma}$ and conversely.

If there exists a facet $\boldsymbol{F}$ of $\boldsymbol{\Gamma}$ satisfying that the dimension of $\boldsymbol{F} \cap \boldsymbol{\Gamma}_{1}$ is smaller than or equal to $n-2$, then, by applying Step 1 in the general iteration of the algorithm 
discussed above, on the facet $\boldsymbol{F}$ with $\boldsymbol{F} \cap\left\{p^{1}, \ldots, p^{t}\right\}$ as the set of extreme points on it determined already, we can find a new extreme point on $F$ which is not in $\boldsymbol{\Gamma}_{1}$. On the other hand, if all the facets $\boldsymbol{F}$ of $\boldsymbol{\Gamma}$ are such that $\boldsymbol{F} \cap \boldsymbol{\Gamma}_{1}$ has dimension $n-1$, then $\boldsymbol{F} \cap \boldsymbol{\Gamma}_{1}$ is a segment of the facet $\boldsymbol{F}$ for every facet of $\boldsymbol{\Gamma}$. In this case it seems reasonable to conjecture that $\left\langle\left(D_{1}\right)^{\mathrm{T}}, \ldots,\left(D_{m_{1}}\right)^{\mathrm{T}}\right\rangle$, the convex hull of the coefficient vectors of the facetal hyperplanes of $\boldsymbol{\Gamma}$, is not a segment of order 1 or 2 of the dual polytope of $\boldsymbol{\Gamma}_{1}$. We give a precise statement of this conjecture now.

Conjecture 5.1. Let $\Gamma$ be the full-dimensional convex polytope in the $y$-space (i.e., $\mathbb{R}^{n}$ ) defined by (14) with each constraint there defining one of its facets as in (15). Let $\left\{p^{1}, \ldots, p^{t}\right\}$ be a subset of extreme points of $\boldsymbol{\Gamma}$ whose convex hull $\boldsymbol{\Gamma}_{1}$ is a segment of order 1 of $\boldsymbol{\Gamma}$. If $\boldsymbol{\Gamma}_{1} \neq \boldsymbol{\Gamma}$, either

(i) there exists at least one facet $\boldsymbol{F}$ of $\boldsymbol{\Gamma}$ such that $\boldsymbol{F} \cap \boldsymbol{\Gamma}_{1}$ has dimension smaller than or equal to $n-2$ (i.e., equivalently, for at least one $i$ between 1 and $m_{1}$, the affine rank of the set of points among $\left\{p^{1}, \ldots, p^{t}\right\}$ satisfying $D_{i} \cdot y=1$ is smaller than or equal to $(n-2))$, or

(ii) for each $i=1, \ldots, m_{1},\left(D_{i} .\right)^{\mathrm{T}}$ is a vector representing a facet of $\boldsymbol{\Gamma}_{1}$, i.e., it is an extreme point of the set of feasible solutions of the following system in terms of the variables $a=\left(a_{1}, \ldots, a_{s}\right)^{\mathrm{T}}$ :

$$
\left(p^{k}\right)^{\mathrm{T}} a \leqslant 1, \quad k=1, \ldots, t,
$$

and $\left\langle\left(D_{1} .\right)^{\mathrm{T}}, \ldots,\left(D_{m_{1}}\right)^{\mathrm{T}}\right\rangle$ is not a segment of order 2 of this set.

Notice that (16) defines the polar dual of $\boldsymbol{\Gamma}_{1}$. If this conjecture is true, it is possible to modify the above algorithm into one for enumerating the extreme points, whose time complexity is bounded above by a polynomial in the unknown number of extreme points.

The second strategy tries to generate extreme points in such a way that the list of known extreme points is never a segment until all the extreme points of the polytope are in the list. For this we are investigating the following conjecture.

Conjecture 5.2. Consider the convex polytope $\boldsymbol{K}$ defined by (1). Let $\left\{d^{1}, \ldots, d^{r}\right\}$ be the set of known extreme points of $\boldsymbol{K}$ such that their convex hull $\boldsymbol{K}_{1}$ has the same dimension as $\boldsymbol{K}$, but is not a segment of $\boldsymbol{K}$. Now identify all the pairs of extreme points among $\left\{d^{1}, \ldots, d^{r}\right\}$ that are adjacent on $\boldsymbol{K}_{1}$ but not on $\boldsymbol{K}$. For each such pair, find the vector $c$ satisfying the condition similar to that in (3). Obtain a new extreme point of $\boldsymbol{K}$ by maximizing $c x$ as in Step 2 of the general iteration of the algorithm discussed above and include it in $\boldsymbol{K}_{1}$. Let $\boldsymbol{K}_{2}$ be the set of known extreme points of $\boldsymbol{K}$ after this process is completed. Then either $\boldsymbol{K}_{2}=\boldsymbol{K}$, or it is not a segment of $\boldsymbol{K}$.

Again, if Conjecture 5.2 is true, we can generate extreme points of $\boldsymbol{K}$ in batches, keeping the nonsegment property of the set of known extreme points at each stage, until all the extreme points are enumerated. 


\section{Acknowledgements}

We are very grateful to Santosh N. Kabadi and Walter D. Morris for many valuable discussions, and to two anonymous referees for valuable suggestions.

\section{References}

[1] W. Altherr, "An algorithm for enumerating all vertices of a convex polyhedron," Computing 15 (1975) $181-183$.

[2] D. Avis and K. Fukuda, "A pivoting algorithm for convex hulls and vertex enumeration of arrangements and polyhedra," Discrete and Computational Geometry 8 (1992) 295-313.

[3] M.L. Balinski, "An algorithm for finding all vertices of convex polyhedral sets," SIAM Journal on Applied Mathematics 9 (1961) 72-88.

[4] C.A. Burdet, "Generating all the faces of a polyhedron," SIAM Journal on Applied Mathematics 26 (1974) 479-489.

[5] M.E. Dyer, "The complexity of vertex enumeration methods," Mathematics of Operations Research 8 (1983) 381-402.

[6] M.E. Dyer and L.G. Proll, "An algorithm for determining all extreme points of a convex polytope," Mathematical Programming 12 (1977) 81-96.

[7] R. Freund and J. Orlin, "On the complexity of four polyhedral containment problems," Mathematical Programming 33 (1985) 139-145.

[8] K. Fukuda and V. Rosta, "Combinatorial face enumeration in convex polytopes," Research Report 93-1, Graduate School of Systems Management, University of Tsukuba (1993).

$19]$ M. Manas and J. Nedoma, "Finding all vertices of a convex polyhedron," Numerische Mathematik 12 (1968) 226-229.

[10] T.H. Matheiss and D.S. Rubin, "A survey and comparison of methods for finding all vertices of convex polyhedral sets," Mathematics of Operations Research 5 (1980) 167-185.

[11] W.D. Morris, Private communication, 1992.

[12] T.S. Motzkin, H. Raiffa, G.L. Thompson and R.M. Thrall, "The double description method," in: H.W. Kuhn and A.W. Tucker, eds., Contributions to the Theory of Games, Vol. II, Annals of Mathematics Studies 28 (Princeton University Press, Princeton, NJ, 1953) pp. 81-103.

[13] K.G. Murty, "The graph of an abstract polytope," Mathematical Programming 4 (1973) 336-346.

[14] K.G. Murty, Linear Programming (Wiley, New York, 1983).

[15] K.G. Murty, "Faces of a polyhedron," Mathematical Programming Study 24 (1985) 219-224.

[16] J.S. Provan, "Efficient enumeration of the vertices of polyhedra associated with network LPs," Technical Report, Department of Operations Research, University of North Carolina (Chapel Hill, NC, 1991).

[17] G. Swart, "Finding the convex hull facet by facet," Journal of Algorithms 6 (1985) 17-48. 